\title{
Congruence Relations on Lattice Implication Algebras
}

\author{
Yi Liu (Corresponding author) \\ Key Laboratory of Numerical Simulation in the Sichuan Provincial College, NeiJiang Normal University \\ Neijing, Sichuan 641112, China E-mail: liuyiyl@126.com
}

Yang Xu

Intelligent Control Development Center, Southwest Jiaotong University

Chengdu, Sichuan 610031, China

Tel: 86-28-8760-0760 E-mail: xuyang@ home.swjtu.edu.cn

Ya Qin

College of Mathematics and Information Science, Neijiang Normal University

Neijiang, Sichuan 641112, China

Tel: 86-832-2343-417 E-mail: qinyaqy@126.com

Chengxi Liu

College of Mathematics and Information Science, Neijiang Normal University

Neijiang, Sichuan 641112, China

Received: October 8, 2010 Accepted: October 26, 2010 doi:10.5539/jmr.v3n2p17

This work is supported by National Natural Science Foundation of P.R.China (Grant no. 60875034).

\begin{abstract}
Lattice implication algebra is an important logic algebra, congruence relations is one of important contents in it. The basic properties and the structures of general congruence relations on lattice implication algebras are discussed; The results that a lattice implication algebra is congruence-permutable is obtained.
\end{abstract}

Keywords: Lattice implication algebra, Congruence relations, Permutable lattice implication algebra

\section{Introduction}

Lattice-valued logic, one of important many-valued logic, it plays an important role for two aspects: One is that it extends the chain-type truth-valued field of some well known present logic to some relatively general lattice. The other is that the incompletely comparable property of truth value characterized by general lattice can more efficiently reflect the uncertainty of human being's thinking, judging and decision. Hence, lattice-valued logic is becoming a research field which strongly influences the development of algebraic logic, computer science and artificial intelligent technology. V. Novak(V.Novak, 1982)research on the lattice-valued logic formal systems.

In order to provide a logical foundation for uncertain information processing theory, especially for the fuzziness, the incomparability in uncertain information in the reasoning, and, establish a logical system with truth value in a relatively general lattice, $\mathrm{Xu}(\mathrm{Y} . \mathrm{Xu}, 1993)$ proposed the concept of lattice implication algebra, and discussed some of their properties in (Y. Xu, etc, 2003; Y.Xu, 1993). Filters and congruence relation are important tools when a algebra is studied. They can give the foundation for logical systems from the semantic viewpoint. Song and $\mathrm{Xu}$, Chang and $\mathrm{Xu}(\mathrm{Z}$.M.Song and Y.Xu, 1997; Z. Y. Chang, Y. Xu, 2007) researched the congruence relations in lattice implication algebras and the relationship between congruence relation and filters, and congruence relation induced by filters and LI-ideals, respectively. Permutable congruence in universal algebra is proposed in (S. Burris, H. P. Sankappanavar, 1981). In view of universal algebra, the conditions of permutable congruence in implication algebra are studied by D. N. Castano and J. P. D. Varela(D. N. Castano, J. P. D. Varela, 2009). In many literatures, many authors studied the congruence relations on an algebra. But, most of the literatures on congruence relations concentrate over the quotient structure induced by congruence relation, not on the general properties.

In this paper, as a continuation of research on congruence relation theory in lattice implication algebras. Firstly, we mainly discussed the basic properties and the structures of general congruence relations on lattice implication algebras. We obtain that a lattice implication algebra is congruence-permutable. 


\section{Preliminaries}

Definition 2.1(Y.Xu, 1993) Let $(L, \vee, \wedge, O, I)$ be a bounded lattice with an order-reversing involution ', the greatest element $I$ and the smallest element $O$, and $\rightarrow: L \times L \longrightarrow L$ be a mapping. $\mathscr{L}=\left(L, \vee, \wedge,{ }^{\prime}, \rightarrow, O, I\right)$ is called a lattice implication algebra if the following conditions hold for any $x, y, z \in L$ :

$\left(I_{1}\right) x \rightarrow(y \rightarrow z)=y \rightarrow(x \rightarrow z)$.

$\left(I_{2}\right) x \rightarrow x=I$.

$\left(I_{3}\right) x \rightarrow y=y^{\prime} \rightarrow x^{\prime}$.

$\left(I_{4}\right) x \rightarrow y=y \rightarrow x=I$ implies $x=y$.

$\left(I_{5}\right)(x \rightarrow y) \rightarrow y=(y \rightarrow x) \rightarrow x$.

$\left(l_{1}\right)(x \vee y) \rightarrow z=(x \rightarrow z) \wedge(y \rightarrow z)$.

$\left(l_{2}\right)(x \wedge y) \rightarrow z=(x \rightarrow z) \vee(y \rightarrow z)$.

In this paper, denote $\mathscr{L}$ as lattice implication algebra $\left(L, \vee, \wedge,{ }^{\prime}, \rightarrow, O, I\right)$.

We list some basic properties of lattice implication algebras. It is useful to develop these topics in other sections.

Theorem 2.2 (Y. Xu, etc, 2003) Let $\mathscr{L}$ be a lattice implication algebra. Then for any $x, y, z \in L$, the following conclusions hold:

(1) $(x \rightarrow y) \rightarrow((y \rightarrow z) \rightarrow(x \rightarrow z))=I$.

(2) if $x \leq y$, then $x \rightarrow z \geq y \rightarrow z$ and $z \rightarrow x \leq z \rightarrow y$.

(3) $(z \rightarrow x) \rightarrow(z \rightarrow y)=(x \wedge z) \rightarrow y=(x \rightarrow z) \rightarrow(x \rightarrow y)$.

(4) $x \rightarrow(y \rightarrow z)=(x \vee y) \rightarrow z$ if and only if $x \rightarrow(y \rightarrow z)=x \rightarrow z=y \rightarrow z$.

(5) $z \leq y \rightarrow x$ if and only if $y \leq z \rightarrow x$.

Definition 2.3 (Z.M.Song, Y. Xu, 1997) Let $\mathscr{L}$ be a lattice implication algebra. $\equiv_{\theta} \subseteq L \times L$ is said to be a congruence relation on $\mathscr{L}$, if $\equiv_{\theta}$ satisfies: for any $x, y, z \in L$

(1) $x \equiv_{\theta} x$.

(2) $x \equiv_{\theta} y$ implies $y \equiv_{\theta} x$.

(3) $x \equiv_{\theta} y, y \equiv_{\theta} z$ implies $x \equiv_{\theta} z$.

(4) $x \equiv_{\theta} y$ implies $x \rightarrow z \equiv_{\theta} y \rightarrow z$.

Definition 2.3 shows that $\equiv_{\theta}$ is an equivalence relation on $L$, and is compatible w.r.t. $\rightarrow$. In [4], define $\otimes, \oplus$ as follows: $x \otimes y=\left(x \rightarrow y^{\prime}\right)^{\prime}$ and $x \oplus y=x^{\prime} \rightarrow y$. Theorem 2.4 will show that is compatible w.r.t. $\vee, \wedge,,^{\prime}, \otimes, \oplus £$

Theorem 2.4 (Z.M.Song, Y. Xu, 1997; Z. Y. Chang, Y. Xu, 2007) If $\equiv_{\theta}$ is a congruence relation on a lattice implication algebra, then for any $x, y, z \in L$,

(1) $x \equiv_{\theta} y$ if and only if $z \rightarrow x \equiv_{\theta} z \rightarrow y$.

(2) $x \equiv_{\theta} y$ if and only if for any $x \vee z \equiv_{\theta} y \vee z$.

(3) $x \equiv_{\theta} y$ if and only if for any $x \wedge z \equiv_{\theta} y \wedge z$.

(4) $x \equiv_{\theta} y$ if and only if $x \otimes z \equiv_{\theta} y \otimes z$.

(5) $x \equiv_{\theta} y$ if and only if $x \oplus z \equiv_{\theta} y \oplus z$.

In this paper, denote $\mathrm{F}(\mathscr{L})$ and $\mathrm{CR}(\mathscr{L})$ as the set of all congruence relations and set of all filters on lattice implication algebra $\mathscr{L}$, respectively.

Theorem 2.5 (Y.Xu,etc,2003) Let $\mathscr{L}$ be a lattice implication algebra. Then the following conditions hold for any $x, y, z \in$ $L$,

(1) $x \otimes(x \rightarrow y)=x \wedge y$.

(2) $x \rightarrow(y \rightarrow z)=(x \otimes y) \rightarrow z$.

(3) $x \rightarrow(x \otimes y)=x \wedge y$. 


\section{Congruence Relations on Lattice Implication Algebras}

\subsection{Properties of congruence relations}

Theorem 3.1 Let $\mathscr{L}$ be a lattice implication algebra. Then

$$
\equiv_{\theta}=\left\{(x, y) \in L \times L \mid x \rightarrow y, y \rightarrow x \in[I]_{\theta}\right\}
$$

for any $\equiv_{\theta} \in \mathrm{CR}(\mathscr{L})$.

Proof For any $(x, y) \in \equiv_{\theta}$, then $x \equiv_{\theta} y$, hence $x \rightarrow y \equiv_{\theta} I$ and $y \rightarrow x \equiv_{\theta} I$, that is, $x \rightarrow y \in[I]_{\theta}$ and $y \rightarrow x \in[I]_{\theta}$.

Conversely, for any $x, y \in L$ and $x \rightarrow y \in[I]_{\theta}, y \rightarrow x \in[I]_{\theta}$, then

$$
\begin{aligned}
x=I \rightarrow x & \equiv_{\theta}(y \rightarrow x) \rightarrow x \\
& =(x \rightarrow y) \rightarrow y \\
& \equiv_{\theta} I \rightarrow y=y
\end{aligned}
$$

that is, $x \equiv_{\theta} y$. Hence $(x, y) \in \equiv_{\theta}$.

Theorem 3.2 Let $\mathscr{L}$ be a lattice implication algebra. Then

$$
\equiv_{\theta_{1}} \subseteq \equiv_{\theta_{2}} \text { if and only if }[I]_{\theta_{1}} \subseteq[I]_{\theta_{2}}
$$

for any $\equiv_{\theta_{1}}, \equiv_{\theta_{2}} \in \mathrm{CR}(\mathscr{L})$.

Proof Suppose that $\equiv_{\theta_{1}} \subseteq \equiv_{\theta_{2}}$. For any $x \in[I]_{\theta_{1}}$, that is, $x \equiv_{\theta_{1}} I$, it follows that $(x, I) \in \equiv_{\theta_{1}} \subseteq \equiv_{\theta_{2}}$. Then $(x, I) \in \equiv_{\theta_{2}}$, that is, $x \equiv_{\theta_{2}} I$. Therefore, $x \in[I]_{\theta_{2}}$. Hence $[I]_{\theta_{1}} \subseteq[I]_{\theta_{2}}$.

Conversely, for any $(x, y) \in \equiv_{\theta_{1}}$, we have that $y \rightarrow x \in[I]_{\theta_{1}}$ and $x \rightarrow y \in[I]_{\theta_{1}}$. Since $[I]_{\theta_{1}} \subseteq[I]_{\theta_{2}}$, so $y \rightarrow x \in[I]_{\theta_{2}}$ and $x \rightarrow y \in[I]_{\theta_{2}}$. It follows that $(x, y) \in \equiv_{\theta_{2}}$ by Theorem 3.1. That is, $\equiv_{\theta_{1}} \subseteq \equiv_{\theta_{2}}$.

Let $\mathscr{L}$ be a lattice implication algebra and $F_{1}, F_{2} \in \mathrm{F}(\mathscr{L}) . \equiv_{F_{1}}, \equiv_{F_{2}}$ are defined as follows: for any $x, y \in L$

$$
\begin{aligned}
& x \equiv_{F_{1}} y \text { if and only if } x \rightarrow y, y \rightarrow x \in F_{1} \\
& x \equiv_{F_{2}} y \text { if and only if } x \rightarrow y, y \rightarrow x \in F_{2} .
\end{aligned}
$$

Therefore, $\equiv_{F_{1}}, \equiv_{F_{1}} \in \mathrm{CR}(\mathscr{L})$ and $[I]_{F_{1}}=F_{1},[I]_{F_{2}}=F_{2}$. So we can obtain the following corollary:

Corollary 3.3 Let $\mathscr{L}$ be a lattice implication algebra and $F_{1}, F_{2} \in \mathrm{F}(\mathscr{L}), \equiv_{F_{1}}, \equiv_{F_{2}} \in \mathrm{CR}(\mathscr{L})$. Then

$$
F_{1} \subseteq F_{2} \text { if and only if } \equiv_{F_{1}} \subseteq \equiv_{F_{2}} .
$$

Theorem 3.4 Let $\mathscr{L}$ be a lattice implication algebra and $\equiv_{\theta_{i}} \in \mathrm{CR}(\mathscr{L})(i \in J)$, where $J$ is an index set. Put $\equiv_{\theta}=\cap_{i \in J} \equiv_{\theta_{i}}$. Then

(1) $\equiv_{\theta} \in \mathrm{CR}(\mathscr{L})$.

(2) $[I]_{\theta}=\cap_{i \in J}\left([I]_{\theta_{i}}\right)$.

Proof (1) By the Definition of congruence relations of $\mathscr{L}$, it is easy to obtain the result.

(2) Since $\equiv_{\theta}=\cap_{i \in J} \equiv_{\theta_{i}}$ for any $i \in J$, it follows that $\equiv_{\theta} \subseteq \equiv_{\theta_{i}}$. By Theorem 3.2, we have $[I]_{\theta} \subseteq[I]_{\theta_{i}}$ for any $i \in J$. Therefore

$$
[I]_{\theta} \subseteq \cap_{i \in J}\left([I]_{\theta_{i}}\right) .
$$

Conversely, for any $x \in \cap_{i \in J}\left([I]_{\theta_{i}}\right)$, then $x \in[I]_{\theta_{i}}$ for any $i \in J$. That is, $(x, I) \in \equiv_{\theta_{i}}(i \in J)$. Therefore $(x, I) \in \cap_{i \in J} \equiv_{\theta_{i}}=\equiv_{\theta}$, that is, $x \in[I]_{\theta}$. Hence

$$
\cap_{i \in J}\left([I]_{\theta_{i}}\right) \subseteq[I]_{\theta}
$$

So the result holds.

Definition 3.5 (Y. Xu, etc, 2003) Let $f$ be a mapping from lattice implication algebra $\mathscr{L}_{1}$ to $\mathscr{L}_{2}$. If

$$
\begin{aligned}
f(x \rightarrow y) & =f(x) \rightarrow f(y) ; \\
f(x \vee y) & =f(x) \vee f(y) ; \\
f(x \wedge y) & =f(x) \wedge f(y) ; \\
f\left(x^{\prime}\right) & =(f(x))^{\prime} .
\end{aligned}
$$


Then $f$ is called a lattice implication homomorphism from $\mathscr{L}_{1}$ to $\mathscr{L}_{2}$.

Theorem 3.6 Let $f$ is a lattice implication homomorphism from $\mathscr{L}_{1}$ to $\mathscr{L}_{2}$. Put

$$
\operatorname{Ker}^{*}(f)=\left\{(a, b) \in L_{1} \times L_{1} \mid f(a)=f(b)\right\} .
$$

Then $\operatorname{Ker}^{*}(f)$ is a congruence relation on $\mathscr{L}_{1}$.

Proof Obviously, $\operatorname{Ker}^{*}(f)$ is a equivalence relation.

Suppose $(x, y) \in \operatorname{Ker}^{*}(f)$, so $f(x)=f(y)$. For any $z \in L_{1}$, it follows that

$$
\begin{aligned}
f(y \rightarrow z) & =f(y) \rightarrow f(z) \\
& =f(x) \rightarrow f(z) \\
& =f(x \rightarrow z)
\end{aligned}
$$

That is, $(x \rightarrow z, y \rightarrow z) \in \operatorname{Ker}^{*}(f)$. Therefore, $\operatorname{Ker}^{*}(f)$ is a congruence relation on $\mathscr{L}$.

Definition 3.7 (Y.Q.Zhu,2008) A lattice implication algebra $\mathscr{L}$ is called simple, if $\{I\}$ and $L$ are the only filters of $\mathscr{L}$.

Theorem 3.8 Let $\mathscr{L}$ be a simple lattice implication algebra. Then $\{(x, x) \mid x \in L\}$ and $L \times L$ are the only congruence relations on $\mathscr{L}$.

Proof Let $\equiv_{\theta} \in \mathrm{CR}(\mathscr{L})$, then $[I]_{\theta}$ is a filter of $\mathscr{L}$. Since $\mathscr{L}$ is a simple lattice implication algebra, then $[I]_{\theta}=\{I\}$ or $[I]_{\theta}=L$.

If $[I]_{\theta}=\{I\}$, then $\equiv_{\theta}=\{(x, x) \mid x \in L\}$. In fact, obviously, $\{(x, x) \mid x \in L\}$ is a congruence relation on $\mathscr{L}$. For any $(x, y) \in \equiv_{\theta}$, then $x \equiv_{\theta} y$, we have $x \rightarrow y \in[I]_{\theta}$ and $y \rightarrow x \in[I]_{\theta}$, therefore $x=y$, that is, $(x, y) \in\{(x, x) \mid x \in L\}$. Conversely, for any $(x, y) \in\{(x, x) \mid x \in L\}$, it is easy to obtain $(x, y) \in \equiv_{\theta}$ by Theorem 2.4.

If $[I]_{\theta}=L$, then $\equiv_{\theta}=L \times L$.

\subsection{Lattice implication algebra is congruence-permutable}

In universal algebra, the concept of two congruence relations on universal algebra are permutable is defined. Let $A$ be an universal algebra, $\equiv_{\theta_{1}}$ and $\equiv_{\theta_{2}}$ be two congruence relations. $\equiv_{\theta_{1}}$ and $\equiv_{\theta_{2}}$ are permutable if

$$
\equiv_{\theta_{1}} \circ \equiv_{\theta_{2}}=\equiv_{\theta_{2}} \circ \equiv_{\theta_{1}}
$$

Universal algebra $A$ is congruence-permutable if every pair of congruencies on $A$ permutes.

In this section, we will prove that a lattice implication algebra is congruence-permutable.

Theorem 3.8 Let $\mathscr{L}$ be a lattice implication algebra. For any $\equiv_{\theta} \in \mathrm{CR}(\mathscr{L})$, then for any $x \in L$, we have that

$$
[x]_{\theta}=\left\{\alpha_{1} \otimes\left(\alpha_{2} \rightarrow x\right) \mid \alpha_{i} \in[I]_{\theta}, i=1,2\right\} .
$$

Equivalently, $y \in[x]_{\theta}$ if and only if there exist $\alpha_{1}, \alpha_{2} \in[I]_{\theta}$ such that

$$
y=\alpha_{1} \otimes\left(\alpha_{2} \rightarrow x\right) .
$$

Proof Let $y \in[x]_{\theta}$, then $x \rightarrow y \equiv_{\theta} I$ and $y \rightarrow x \equiv_{\theta} I$. Put $\alpha_{1}=x \rightarrow y$ and $\alpha_{2}=y \rightarrow x$. Hence $\alpha_{i} \in[I]_{\theta}, i=1,2$. Since

$$
\begin{aligned}
\alpha_{2} \rightarrow x & =(y \rightarrow x) \rightarrow x \\
& =(x \rightarrow y) \rightarrow y \\
& =\alpha_{1} \rightarrow y .
\end{aligned}
$$

it follows that

$$
\alpha_{1} \otimes\left(\alpha_{2} \rightarrow x\right)=\alpha_{1} \otimes\left(\alpha_{1} \rightarrow y\right)=\alpha_{1} \wedge y
$$

We have $y \leq \alpha_{1}$ by $\alpha_{1}=x \rightarrow y$. Therefore

$$
\alpha_{1} \otimes\left(\alpha_{2} \rightarrow x\right)=\alpha_{1} \wedge y=y
$$

Conversely, suppose there exist $\alpha_{1}, \alpha_{2} \in[I]_{\theta}$ such that $y=\alpha_{1} \otimes\left(\alpha_{2} \rightarrow x\right)$. Since $\alpha_{1} \equiv_{\theta} I$ and $\alpha_{2} \equiv_{\theta} I$, it follows that

$$
\alpha_{2} \rightarrow x \equiv_{\theta} I \rightarrow x=x .
$$


Hence,

$$
\begin{aligned}
\alpha_{1} \otimes\left(\alpha_{2} \rightarrow x\right) & \equiv_{\theta} \alpha_{1} \otimes x \\
& \equiv_{\theta} I \otimes x=x
\end{aligned}
$$

Therefore, $y=\alpha_{1} \otimes\left(\alpha_{2} \rightarrow x\right) \in[x]_{\theta}$.

Definition 3.10 (Y.Xu and K.Y.Qin, 1992) Lattice implication algebra $\mathscr{L}$ is a lattice $H$ implication algebra, if for any $x, y, z \in L$,

$$
x \vee y \vee((x \wedge y) \rightarrow z)=I
$$

Corollary 3.11 Let $\mathscr{L}$ be a lattice $H$ implication algebra. $\mathscr{L}$ is a lattice $H$ implication algebra.For any $\equiv_{\theta} \in \mathrm{CR}(\mathscr{L})$, then for any $x \in L$, we have that

$$
[x]_{\theta}=\left\{\alpha_{1} \wedge\left(\alpha_{2} \rightarrow x\right) \mid \alpha_{i} \in[I]_{\theta}, i=1,2\right\} .
$$

Theorem 3.12 Let $\mathscr{L}$ be a lattice implication algebra and $\equiv_{\theta_{1}}, \equiv_{\theta_{2}} \in \mathrm{CR}(\mathscr{L})$. Then

$$
\equiv_{\theta_{1}} \circ \equiv_{\theta_{2}}=\equiv_{\theta_{2}} \circ \equiv_{\theta_{1}} \text {. }
$$

Proof Let $a \equiv_{\theta_{1}} x \equiv_{\theta_{2}} b$. So $a \in[x]_{\theta_{1}}$ and $b \in[x]_{\theta_{2}}$. By Theorem 3.9, it follows that there exist $\alpha_{1}, \alpha_{2} \in[I]_{\theta_{1}}$ and $\beta_{1}, \beta_{2} \in[I]_{\theta_{2}}$ such that

$$
a=\alpha_{1} \otimes\left(\alpha_{2} \rightarrow x\right) \text { and } b=\beta_{1} \otimes\left(\beta_{2} \rightarrow x\right) .
$$

Since $\beta_{1}, \beta_{2} \in[I]_{\theta_{2}}$, so $\beta_{1} \equiv_{\theta_{2}} I$ and $\beta_{2} \equiv_{\theta_{2}} I$. Thus

$$
\alpha_{1} \wedge \beta_{1} \equiv_{\theta_{2}} \alpha_{1} \wedge I=\alpha_{1}
$$

and

$$
\alpha_{2} \wedge \beta_{2} \equiv_{\theta_{2}} \alpha_{2} \wedge I=\alpha_{2}
$$

Therefore,

$$
\begin{gathered}
\left(\alpha_{2} \wedge \beta_{2}\right) \rightarrow x \equiv_{\theta_{2}} \alpha_{2} \rightarrow x \\
a=\alpha_{1} \otimes\left(\alpha_{2} \rightarrow x\right) \quad \equiv_{\theta_{2}} \alpha_{1} \otimes\left(\left(\alpha_{2} \wedge \beta_{2}\right) \rightarrow x\right) \\
\\
\equiv_{\theta_{2}}\left(\alpha_{1} \wedge \beta_{1}\right) \otimes\left(\left(\alpha_{2} \wedge \beta_{2}\right) \rightarrow x\right) .
\end{gathered}
$$

Since $\alpha_{1}, \alpha_{2} \in[I]_{\theta_{1}}$, that is, $\alpha_{1} \equiv_{\theta_{1}} I$ and $\alpha_{2} \equiv_{\theta_{1}} I$.

$$
\alpha_{1} \wedge \beta_{1} \equiv_{\theta_{1}} \beta_{1} \text { and } \alpha_{2} \wedge \beta_{2} \equiv_{\theta_{1}} \beta_{2} \text {. }
$$

By (3.1) and (3.2), we have that

$$
\begin{array}{ll}
a=\alpha_{1} \otimes\left(\alpha_{2} \rightarrow x\right) \quad & \equiv_{\theta_{2}}\left(\alpha_{1} \wedge \beta_{1}\right) \otimes\left(\left(\alpha_{2} \wedge \beta_{2}\right) \rightarrow x\right) \\
& \equiv_{\theta_{1}} \beta_{1} \otimes\left(\beta_{2} \rightarrow x\right)=b .
\end{array}
$$

That is, there exists $y=\left(\alpha_{1} \wedge \beta_{1}\right) \otimes\left(\left(\alpha_{2} \wedge \beta_{2}\right) \rightarrow x\right)$ such that $a \equiv_{\theta_{2}} y \equiv_{\theta_{1}} b$. Therefore, the theorem holds.

\section{Conclusion}

In order to research the many-valued logical system whose propositional value is given in a lattice, Xu initiated the concept of lattice implication algebras. Hence for development of this many-valued logical system, it is needed to make clear the structure of lattice implication algebras. It is well known that to investigate the structure of an algebraic system, the filters and congruence with special properties play an important role. Firstly, we mainly discussed the basic properties and the structures of general congruence relations on lattice implication algebras. We obtain that a lattice implication algebra is congruence-permutable. Finally, we discussed properties of congruence relations on finite lattice implication algebras. We hope that above work would serve as a foundation for enriching corresponding many-valued logical system.

\section{References}

D. N. Castano, J. P. D. Varela. (2009). Conditions for permutable of congruence in implication algebras. order, 26, 245-254.

J. Pavelka. (1979). On fuzzy logic. Studia Logica, I,II,III, £eit.Math. Logik u. Grundl.Math., 2545-52, 119-134, 447-464.

S. Burris, H. P. Sankappanavar. (1981). A course in universal algebra. Springer-Verlag, New York. 
V. Novak. (1982). First order fuzzy logic. Studia Logica, 46(1), 87-109.

Y. B. Jun, E.H. Roh, Xu Yang. (1998). LI-ideal in lattice implication algebra. J. Bull Korean Math. Soc., 35(1), 13-24.

Y. B. Jun, Y. Xu and K. Y. Qin. (1998). Positive implicative and associative filters of lattice implication algebras. Bull. Korean Math. Soc., 35(1), 53-61.

Y. Q. Zhu. (2008). Finite simple lattice implication algebras. China Quart. J. of Math., 23 (3), 423-429.

Y. Xu and K.Y.Qin. (1992). Lattice H implication algebras and lattice implication algebra classes. J. Hebei Mining and Civil Engineering Institute, 3, 139-143.

Y. Xu, D. Ruan, K. Y. Qin, J. Liu. (2003). Lattice-Valued Logic-An Alternative Approach to Treat Fuzziness and Incomparability. Berlin: Springer-Verlag.

Y. Xu. (1993). Lattice implication algebra. J.Southwest Jiaotong Univ., 28(1), 20-27.

Z. M. Song, Y. Xu. (1997). Congruence relations on lattice implication algebras. J. Math Appl., 10(3), 121-124.

Z. Y. Chang, Y. Xu. (2007). Congruence relations induced by filters and LI-ideals. Theor. Adv. and Appl. of Fuzzy Logic, 10, 349-357. 